\title{
Draft Genome of the Liver Fluke Fasciola gigantica
}

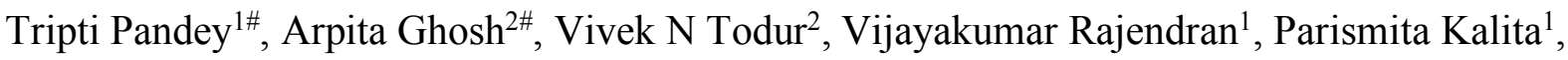
Jupitara Kalita ${ }^{1}$, Rohit Shukla ${ }^{1}$, Purna B Chetri ${ }^{1}$, Harish Shukla ${ }^{1}$, Amit Sonkar ${ }^{1}$, Denzelle Lee Lyngdoh ${ }^{1}$, Radhika Singh ${ }^{1}$, Heena Khan ${ }^{1}$, Joplin Nongkhlaw ${ }^{1}$, Kanhu Charan Das ${ }^{1}$, Timir Tripathi $^{1 *}$

${ }^{1}$ Molecular and Structural Biophysics Laboratory, Department of Biochemistry, North-Eastern Hill University, Shillong 793022, India

${ }^{2}$ Eurofins Genomics India Pvt. Ltd, Doddanekkundi, Bengaluru 560048, India

* Corresponding author: Email: timir.tripathi@gmail.com

${ }^{\#}$ Both authors contributed equally to the work 
Supplementary Figure S1. GO classification of genes similar in F. hepatica and F. gigantica.
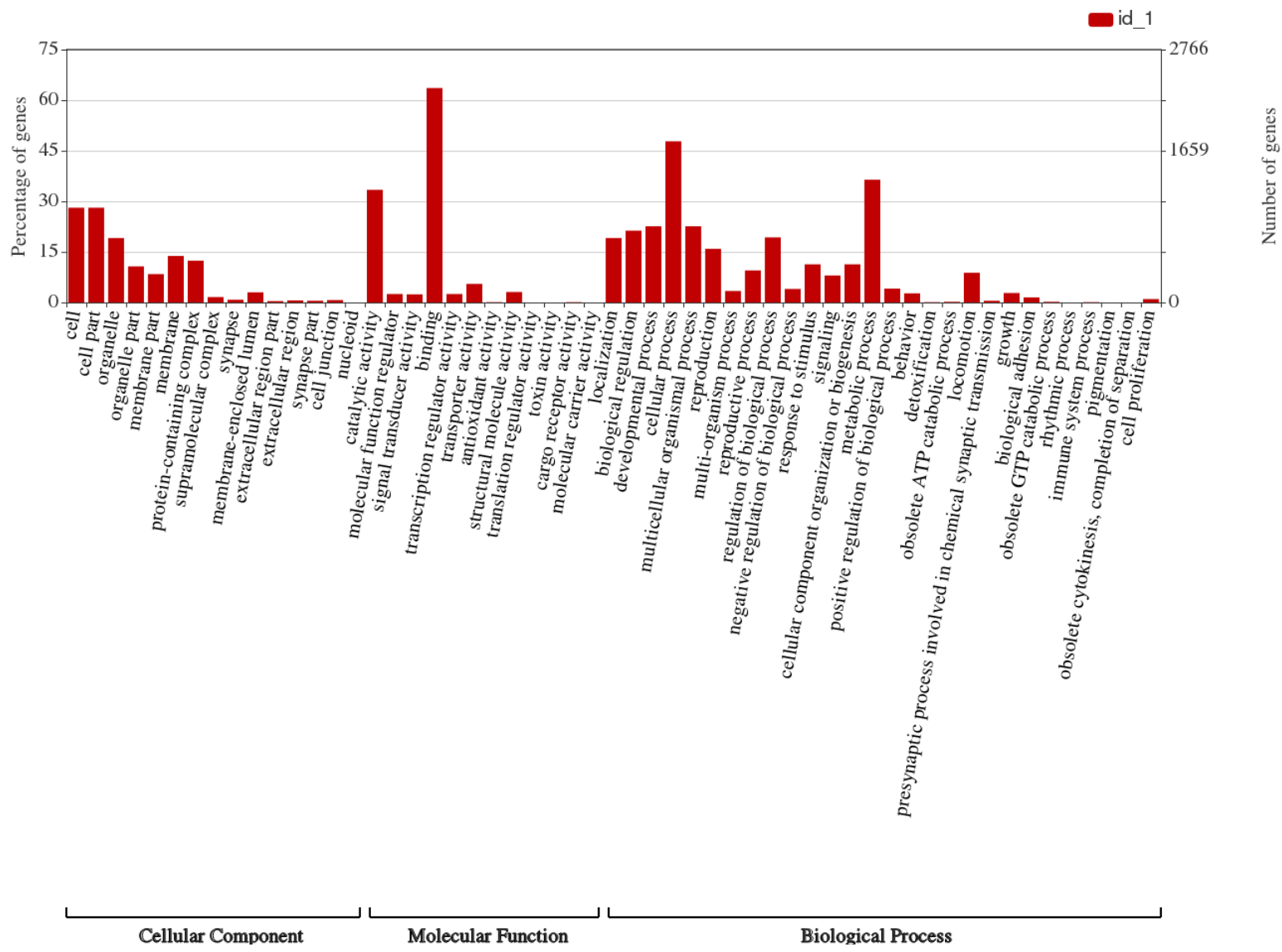
Supplementary Figure S2. Schematic pathway of glycolysis ${ }^{1-3}$.

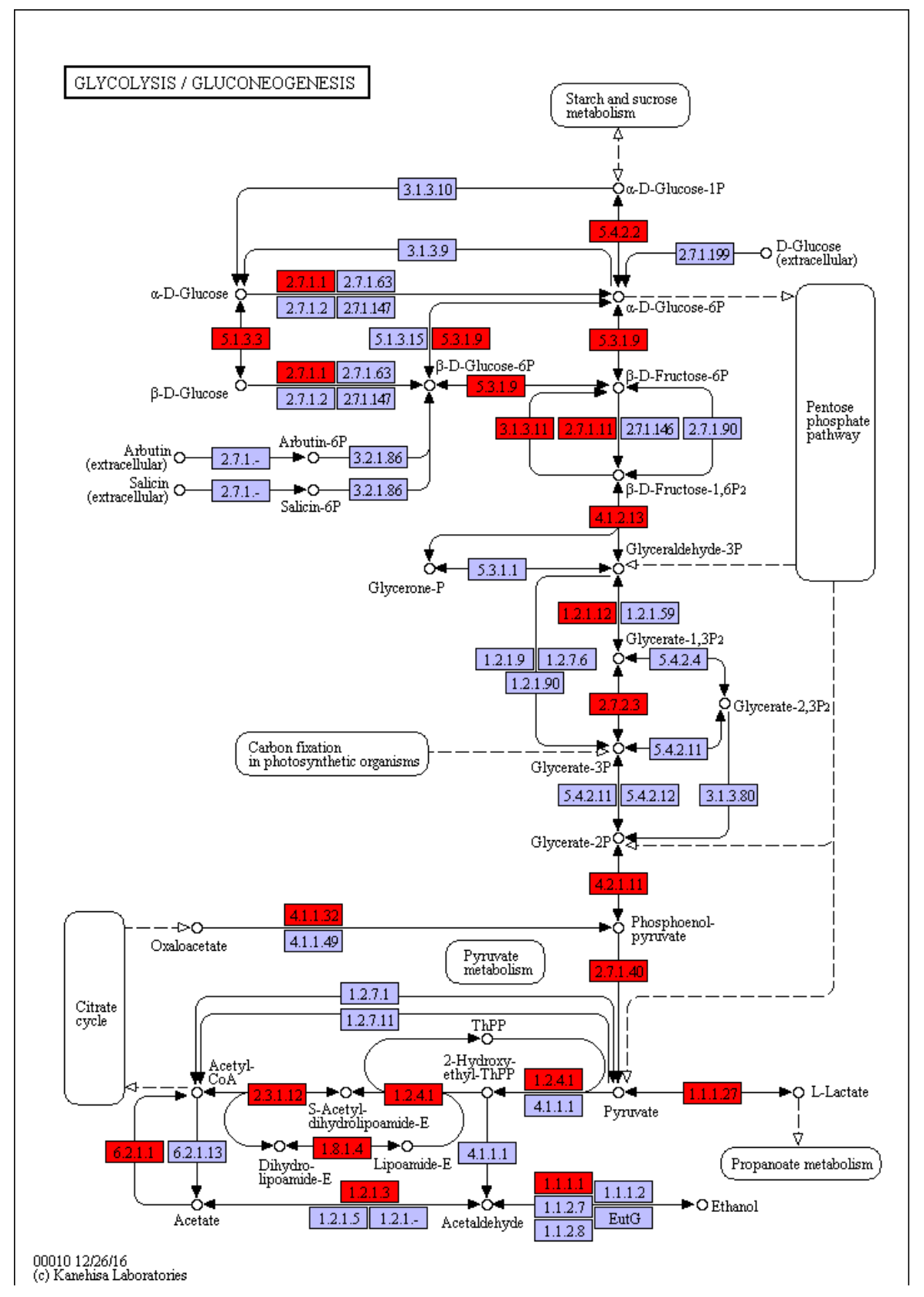


Supplementary Figure S3. Schematic pathway of TCA cycle ${ }^{1-3}$.

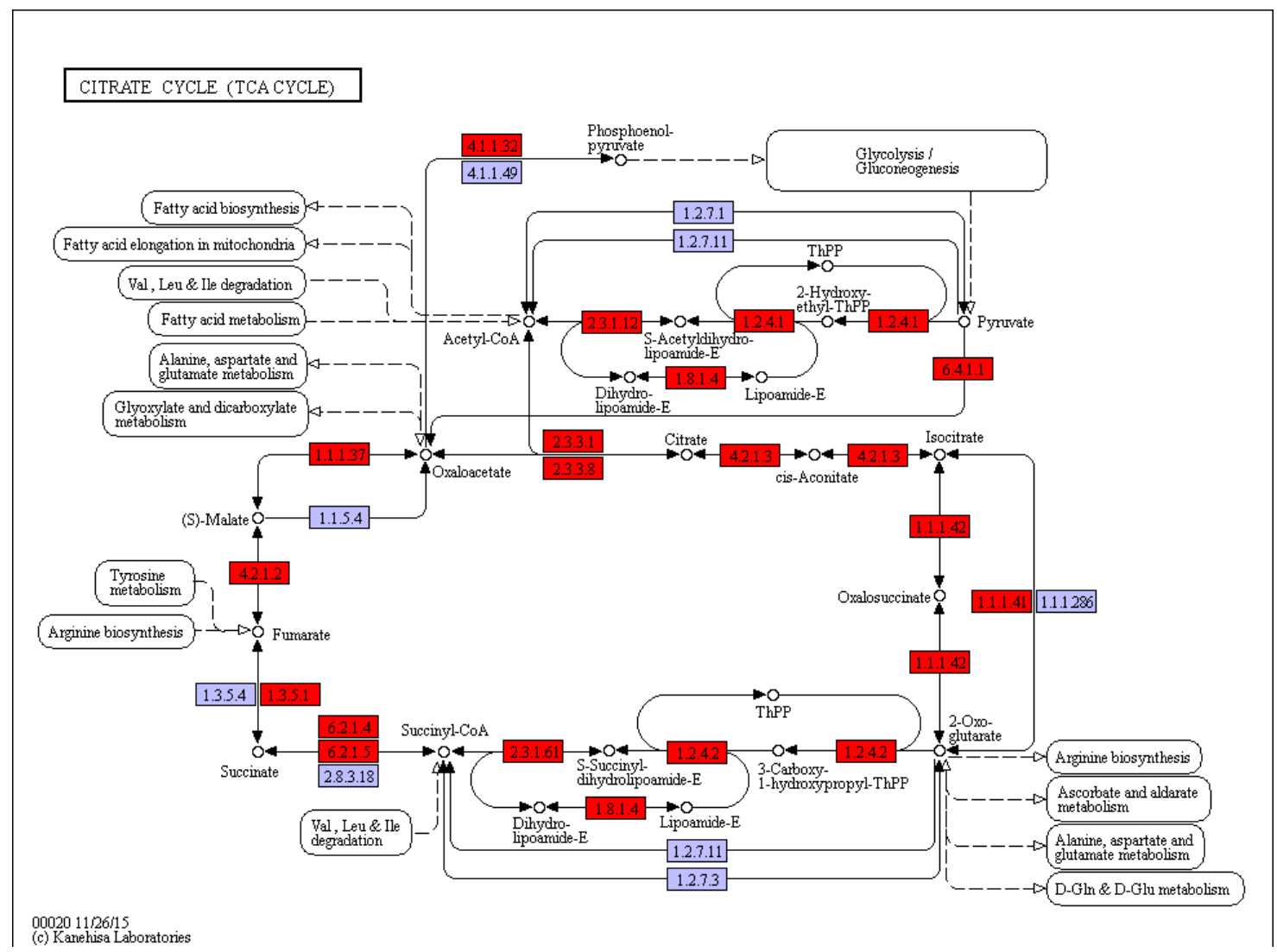


Supplementary Figure S4. Schematic pathway of fatty acid biosynthesis ${ }^{1-3}$.

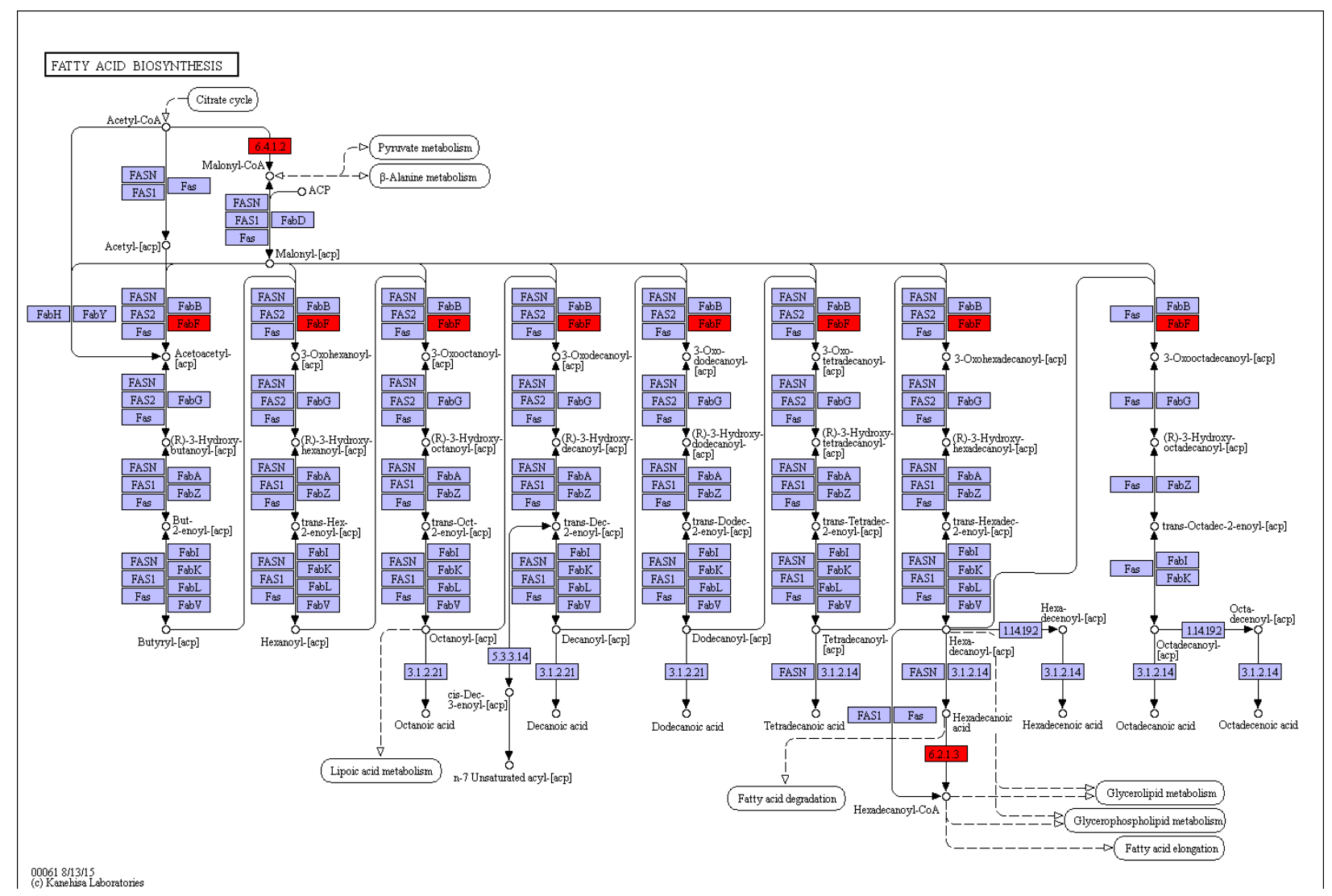


Supplementary Figure S5. Schematic pathway of fatty acid degradation ${ }^{1-3}$.

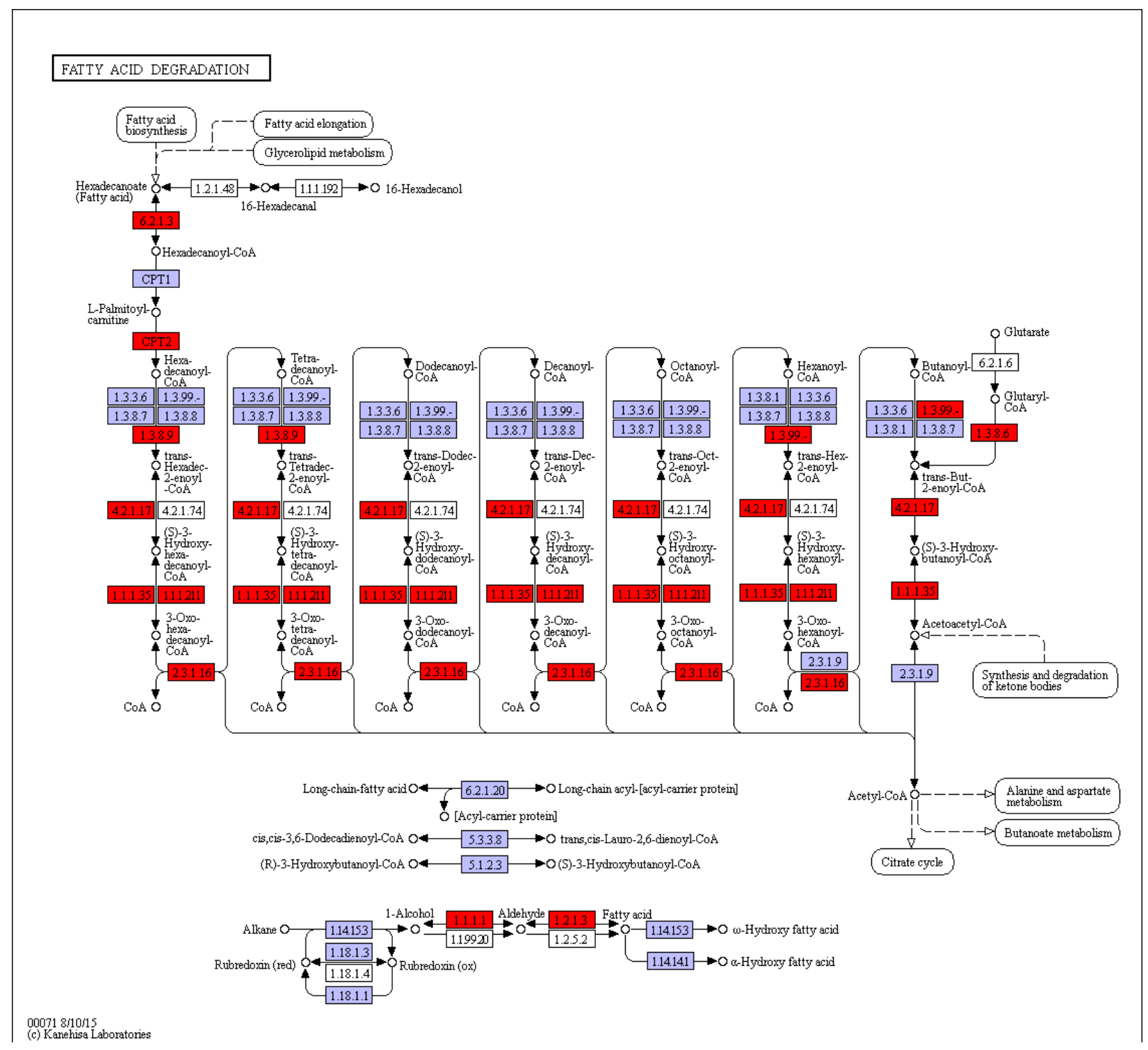




\section{Note:}

The Supplementary Figure S2-S5 are taken from the KEGG website (https://www.genome.jp/kegg/) and are cited at places used, and reference are provided below:

\section{References for KEGG pathways:}

1 Kanehisa, M., Furumichi, M., Tanabe, M., Sato, Y. \& Morishima, K. KEGG: new perspectives on genomes, pathways, diseases and drugs. Nucleic Acids Res 45, D353-361, (2017).

2 Kanehisa, M. \& Goto, S. KEGG: Kyoto encyclopedia of genes and genomes. Nucleic Acids Res 28, 27-30 (2000).

3 Kanehisa, M., Sato, Y., Kawashima, M., Furumichi, M. \& Tanabe, M. KEGG as a reference resource for gene and protein annotation. Nucleic Acids Res 44, D457-462 (2016). 